\title{
INTEGRATED APPROACH FOR OPTIMIZING THE TOURISTIC SPACE PLAN FOR SUSTAINABLE URBAN DEVELOPMENT: A CASE STUDY OF DALIAN CITY, CHINA
}

\author{
QUNSONG ZHANG ${ }^{1,2}$, EUNYOUNY KIM ${ }^{1}$, CUIXIA YANG ${ }^{2}$, FUCUN CAO $^{2}$ \& VAN-NAM HUYNH ${ }^{1}$ \\ ${ }^{1}$ School of Knowledge Science, Japan Advanced Institute of Science and Technology, Japan \\ ${ }^{2}$ Landscape Architecture, School of Art and Design, Dalian Polytechnic University, China
}

\begin{abstract}
As Dalian, China, is a touristic city, optimization of the touristic space plan has become increasingly important for its sustainable development. In recent years, big data, GIS, computer simulation and other technologies have been applied widely in the field of tourism spatial planning research. However, the integration of those information technology-based methods with the sustainable urban development framework has not been sufficiently studied to suggest how to optimize the spatial planning strategies for scenic spots in urban areas. This paper aims to propose a method for optimizing the layout of tourism space based on the sustainable urban development framework. We used five districts, including the centre of Dalian, as the background for the evolution of scenic spots; simultaneously, we used integrated methods including kernel density estimation (KDE) and spatial syntax, to analyse the spatial pattern of tourism in Dalian. We used big data from the three most authoritative evaluation websites in China, to collect the current popularity and location data of scenic spots. According to the analysis of spatiotemporal patterns, and transportation accessibility of each scenic spot, the results show that there are some problems in the network of tourist attractions, such as low connectivity and unbalanced distribution. Therefore, we propose a method for optimizing the geographical views of spatial structure for tourist attractions in Dalian, as a countermeasure of sustainable urban development.

Keywords: touristic space plan, sustainable urban development, spatiotemporal distribution, scenic spot heat, big data.
\end{abstract}

\section{INTRODUCTION}

\subsection{Research background}

Building safe, inclusive and sustainable cities and residential areas has become a global goal, which affects the development direction of each city during the process of urbanization [1]. Sustainable urban development is defined as a collection of simultaneous and coordinated evolutions in each part of the city, including economic, social and environmental aspects [2]. In addition to resolving the contradiction between the types and scale of urban land use, population growth and urban pollution, it is also very important to consider reasonable and forward-looking infrastructure construction, and investment in urban planning that conforms to urban positioning. As Dalian, China, is a touristic city, optimization of the touristic space plan has become increasingly vital for its sustainable development. The definition of tourism space optimization refers not only to the design of the site, but also to applying an approach which involves comprehensive land use planning, collaborative synergy with the government, multidisciplinary application, and respect for the interests of local stakeholders [3], [4]. For tourism cities, the reasonable planning of tourism space will promote sustainable urban development; thus, the two concepts are mutually beneficial and interdependent. Tourism accounts for a large proportion of local fiscal revenue because the government has introduced many measures to encourage the development of local tourism, and vice versa. 
Additionally, the tourism industry provides vast amounts of employment, improves the residents' income and reduces the pay gap, which is beneficial to the local people's livelihood. Thus, the rational development and utilization of tourism's ecological resources can effectively preserve the local environment.

In recent years, big data, geographic information systems (GIS), computer simulation and other technologies have been applied widely in the field of tourism spatial planning research. Moreover, smartphones and travel websites provide strong technical support for smart tourism, due to the progress of social media [5], [6]. There are many forms of big data collection and application, among which visual analysis is frequently used. Spatial and temporal distribution refers to the visualization of collected big data from the perspective of geography. Some scholars have attempted to study tourist destinations from the perspective of geoinformatics, such as tourists' behaviour patterns [7], tourists' footprint [8] and geotagged photos, etc. [9]. Tourism space and hot spot distribution were also analysed by using tourist registration information, TripAdvisor data via social media [10], global positioning (GPS) technology [11], and the areas' function and tourist density.

However, the integration of those information technology methods has not been studied sufficiently to suggest a direction for optimizing the spatial planning strategies for scenic spots in urban areas. Furthermore, many new conundrums have hindered cities in their continuous development of the tourism market. From a macro perspective, the lack of effective supervision of tourism planning by local governments leads to the destruction of the urban environment and imbalanced economic development, resulting in social problems. This paper aims to propose a method for optimizing the layout of tourism space, based on the sustainable urban development framework, and by employing big data, GIS and axis analysis of spatial syntax. As a result, we propose a method for optimizing the geographical views of spatial structure for tourist attractions in Dalian, as a countermeasure of urban sustainable development.

We first constructed a macro summary of Dalian's tourism economy in recent years, from the perspective of tourism economic income (Section 3.1). Secondly, we classified 331 scenic spots from the perspective of their natural and humanistic environment (Section 3.2.1). In order to further explore the spatial structure and hierarchical distribution of the scenic spot scale in Dalian, we expanded the time dimension. In Sections 3.2.2 and 3.2.3, we present the spatial-temporal distribution and the relevant survey results, such as for the four periods of winter vacation, summer vacation, mini-break, and weekend, which are the peak times for tourists to gather and for citizens to travel to tourist destinations. In addition, we conducted a global integration analysis (Section 3.3) to assess the study areas' traffic accessibility. Combined with examining the changes in tourism income and the scenic spot classification, we proposed an optimization strategy for adjusting the tourism consumption and production structure, from the perspective of sustainable economic development. We identified the spatial structure of four different scenic spots, then analysed their spatial-temporal characteristics and regular patterns, using the geographical method. By verifying the degree of spatial integration in urban tourists' preferences, the key to solving the traffic problem was found, and the optimization strategy for tourism destinations was elaborated, from the perspective of sustainable development of the urban society and environment.

\subsection{Research area}

This study was conducted in Dalian, which is a crucial centre city, port and scenic tourist city on the northern coast of China. It is situated on the southern edge of the Liaodong Peninsula, at the junction of the Yellow Sea and the Bohai Sea, between $38^{\circ} 43^{\prime} 40^{\circ} 12^{\prime} \mathrm{N}$ and 
$120^{\circ} 58^{\prime} 123^{\circ} 31^{\prime} \mathrm{E}$. The total land area of the city is $12,573.85 \mathrm{~km}^{2}$, of which the urban area is $2,414.96 \mathrm{~km}^{2}$; the registered population is 5.952 million. As of 2018 , the city has a jurisdiction covering seven districts, one county and two county-level cities. Dalian's basic landform is high in the centre, and descending stepwise towards the east and west sides, with more hills, as shown in Fig. 1. As a city rich in natural resources for tourism, Dalian has a coastline of 2,211 km. Dalian's number of A-level tourist attractions had increased to 51 by 2018, including two at 5A level, 19 at 4A, 19 at 3A and 11 at 2A; the principal part of urban tourism resources is constituted by coastal tourism resources. Simultaneously, Dalian also has a distinctive cultural landscape, with many historical heritage sites and a special urban landscape. In order to provide a more detailed and in-depth study, and to accurately optimize the layout of tourism space in accordance with urban sustainable development, we chose to include five districts of Dalian city centre (see Fig. 1), namely Zhongshan, Xigang, Shahekou, Ganjingzi and Lvshunkou.
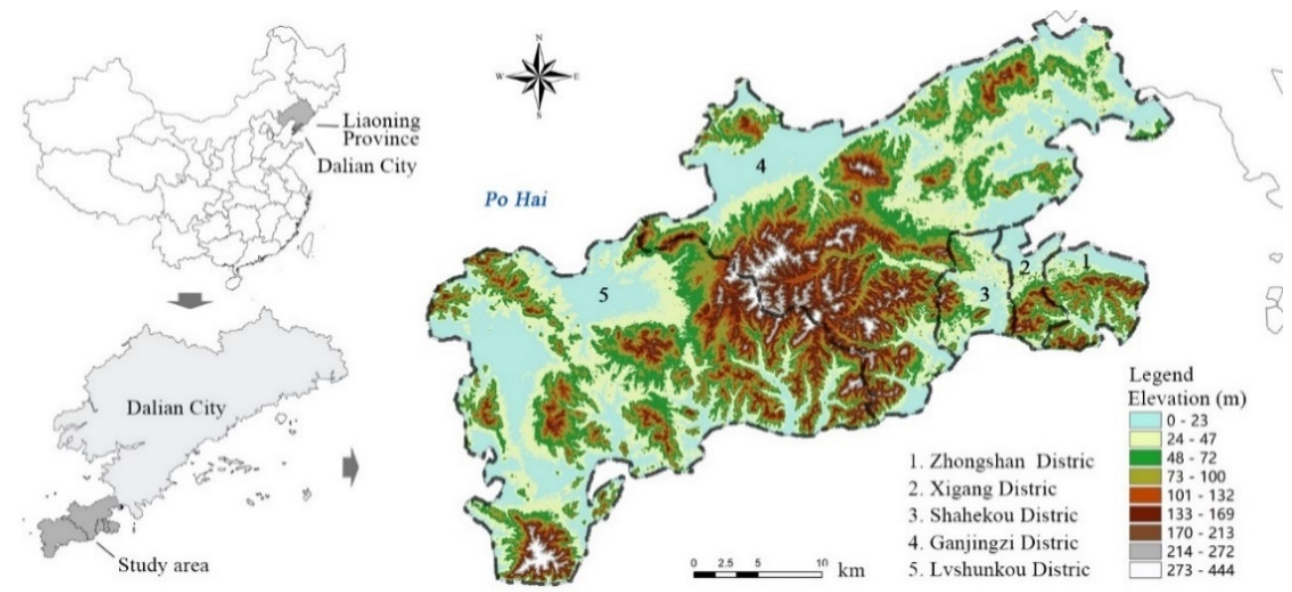

Figure 1: Study area and topographic map of study area.

\section{MATERIALS AND METHODS}

\subsection{Data collection and processing}

Because this paper is conducted within the framework of urban sustainable development, the data we collected mainly included the tourism economy's income, the popularity and spatial distribution of scenic spots, and other relevant data. The economic development data of tourism are obtained from The Statistical Yearbook of Liaoning Province 2018. We collected the foreign exchange income of Dalian from 2013 to 2017, the number of foreign tourists received, the number of local tourists, etc. As a touristic city, the sustainable development of its environment and society is closely related to the types and distribution of tourist attractions. In order to further explore the relationship between these factors, data resources for this research were mainly collected from several authoritative Chinese evaluation websites: Dianping.com, Mafengwo.com and Ctrip.com. Dianping.com is one of the largest guide websites for city life and consumption in China; it is a platform for commenting and sharing information on catering and entertainment, mainly based on tripartite reviews. In addition, Dianping has client software for Android, iPhone and Symbian S60 mobile 
platforms, and can also find scenic spots through GPS. The number of monthly active users has exceeded 250 million by February 2020, mainly distributed in first-tier cities and tourist cities. Mafengwo.com is a travel-based social platform with three core functions: UGC, travel big data, and a free travel trading platform. So far, the hornet's nest has amassed 178 million real user reviews. Ctrip.com is a major travel website in China; it mainly provides customers with vacation product lines, and offers travel consultation and reservation services for guests.

We collected the relevant data for the scenic spots from the three websites. These attractions were acquired from the popularity rankings on these three websites; these rankings were dependent on the number of comments. At least one comment for each attraction is included in the research scope of this paper. Through the forward geocoding service, the address was converted into the mapping coordinates (latitude and longitude); then, duplicate scenic spots were deleted, and changed or abandoned scenic spots were excluded. By 31 January 2020, a total of 331 valid scenic spots had been collected. The original dataset included the user's name, attraction name, attraction category, attraction ranking, location, per capita consumption quota, comment time and time of updating comment, text messages and picture comments, etc. The hot spots in Dalian provided by Ctrip were adjusted according to the season and national holidays, to allow assessment of the hot spots in different periods, and combined with the time analysis of the datasets of the other two websites, to explore the hot spots in different periods.

\subsection{Analysis methods}

Based on the previous section, we took the users' evaluation of the scenic spot as the criterion of its popularity. The date of comments was employed to enable temporal analysis of scenic spots in different periods [12]. We used 10.7 Arc GIS software; the kernel density estimation (KDE) method was adopted to provide the visual analysis [13]; and the grid size was $20 \mathrm{x}$ $20 \mathrm{~m}$. This method is used to express the distribution characteristics of spatial elements by studying the spatial form and variation of distribution density of elements in the area, as this emphasizes the spatial dispersion or aggregation characteristics. In this article, we employed the quartic function

$$
\frac{15}{16}\left(1-t^{2}\right) I(|t|<1)
$$

The kernel density estimation formula we used is eqn (1)

$$
\hat{f}_{h}(x)=\frac{1}{n h} \sum_{i=1}^{n} K\left(\frac{x-x_{i}}{h}\right),
$$

where $x$ is any point in space, $f(x)$ is kernel density at $x, x_{i}$ is all points within the distance $h$ of length $x$, and a weighted superposition of all internal $x_{i}$ operation kernel functions gives the kernel density at $x$.

$h$ stands for default bandwidth; the formula is shown as eqn (2)

$$
\text { SearchRadius }=0.9 * \min \left(S D, \sqrt{\frac{1}{\ln (2)}} * D_{m}\right) * n^{-0.2},
$$

where $S D$ is the standard distance, $D_{m}$ is the median distance. If the population field is not used, $n$ is the number of points; otherwise, $n$ is the sum of the population field values. 
In this paper, simultaneously, the "axis" of space syntax was applied to quantify the street network of Dalian; this indicates the road system of the city, which could be used to analyse and predict the flow of people and vehicles. Therefore, we analysed the syntactic parameters of the global integration value of urban streets in Dalian city, which represented the aggregation or dispersion degree of a spatial unit in relation to other spatial nodes in the system. When the integration value was larger, this meant the node was more accessible in the system, and would gather pedestrian volume more easily.

\section{RESULTS}

\subsection{Tourism economy}

As Dalian is a tourist city, tourism has a substantial impact on the overall development of its economy, as it significantly promotes the development of related industries. According to data from the Statistics Bureau of Liaoning Province and the Statistics Bureau of Dalian City (as shown in Table 1), the number of foreign tourists unexpectedly dropped in 2014; although it then slowly increased (average growth rate $-2.65 \%$ from 2013 to 2017), it was still far from the scale of 2013. The number of domestic tourists rose at an average annual rate of $15.2 \%$. However, with the advance of other tourist coastal cities in China, the regional characteristics of Dalian have gradually weakened. At the same time, the private car blowout has developed. With the reduced proportion of inbound tourism in Dalian, long-distance cross-provincial tours and short-distance tours in surrounding cities and towns have become the main form of tourism.

Table 1: Economic situation of tourism in Dalian. (Source: Statistical Yearbook of Liaoning Province, 2018.)

\begin{tabular}{|l|c|c|c|c|c|}
\hline Indicators & $\mathbf{2 0 1 3}$ & $\mathbf{2 0 1 4}$ & $\mathbf{2 0 1 5}$ & $\mathbf{2 0 1 6}$ & $\mathbf{2 0 1 7}$ \\
\hline $\begin{array}{l}\text { Domestic tourist reception } \\
\text { (million person visits) }\end{array}$ & 52.309 & 56.198 & 68.281 & 76.338 & 84.100 \\
\hline $\begin{array}{l}\text { Domestic tourism income } \\
\text { (billion RMB) }\end{array}$ & 85.04 & 96.53 & 97.72 & 110.52 & 124.29 \\
\hline $\begin{array}{l}\text { Number of inbound tourists } \\
\text { received (person visits) }\end{array}$ & $1,190,035$ & 965,615 & 984,647 & $1,044,100$ & $1,063,938$ \\
\hline $\begin{array}{l}\text { Tourist foreign exchange } \\
\text { income (million USD) }\end{array}$ & 81,341 & 46,012 & 51,625 & 53,948 & 55,081 \\
\hline $\begin{array}{l}\text { The average number of } \\
\text { days visitors stay }\end{array}$ & 3.4 & 3.4 & 3.1 & 3.3 & 3.4 \\
\hline
\end{tabular}

\subsection{Main spatiotemporal patterns and popularity of scenic spots based on environment}

\subsubsection{Classification and distribution of scenic spots}

The classification and integration of scenic spots helped us explore the trends of urban land use and environmental development. We mainly completed the classification of all tourist attractions through the sites' resource characteristics and functions. They were divided into two categories: natural tourist resources and humanities tourist resources. Natural tourist resources were mainly divided into categories of geomorphologic landscape, hydrologic landscape and biological resources; while historic landmarks and sites comprised museums, local culture and arts, and religious culture. Finally, townscape, modern facilities, and 
catering and shopping constituted humanities tourist resources. There were ten sub-types, as shown in Fig. 2(a). It is worth mentioning that although some scenic spots are located at the seaside, they mainly show the fossil remains of geological changes. For instance, the geographic popularization area of Lianhuashan Park is a scenic spot that displays the geomorphologic landscape. In addition, for scenic spots with complex attributes, we used the subjective experience of tourists as a classification criterion. Secondly, many scenic spots are multi-functional and rely on heterogeneous resources, such as theme parks, amusement parks and ecological parks. We determined the types of resources in each park based on its main functions and main facilities.

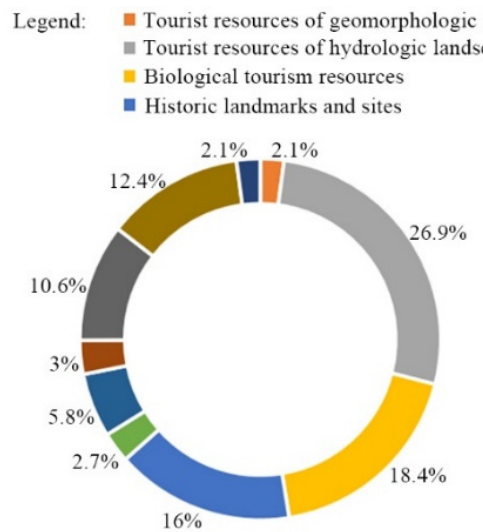

(a)

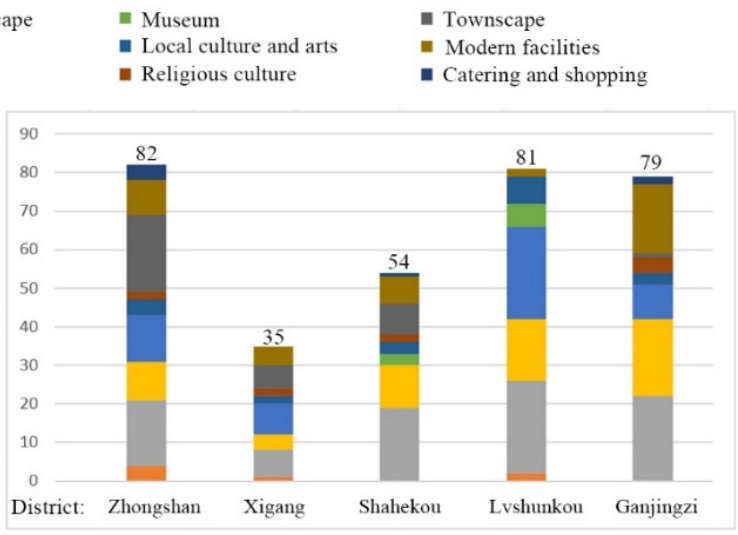

(b)

Figure 2: Sub-types of tourist resources and distribution of scenic spots in Dalian. (a) The proportion of different types of scenic spots; and (b) The proportion of types of scenic spots in different districts.

Simultaneously, we also divided the scenic spots according to the administrative area: as shown in Fig. 2(b), the area with the largest number of scenic spots is Zhongshan, and Xigang has the fewest. However, according to the land area of each district, Zhongshan has the most dense distribution of scenic spots, and Ganjingzi the least. Compared with other districts, there are more scenic spots of townscape types (20) in Zhongshan, which is the district with the most abundant types of scenic spots and the most humanistic tourism resources (51). Another district with more humanistic tourism resources (23) than natural resources (12) is Xigang. Lvshunkou, which is more focused on two types of scenic spots - hydrologic landscapes and historic landmarks and sites - with 24 sites each. This is related to the geographical location at the demarcation line between the Huanghai Sea and Bohai Sea, as well as its beautiful scenery; Lvshunkou is also historically important, as it was established as a military port and was the starting point of Dalian's history. Ganjingzi has the same number of natural resource scenic spots as Lvshunkou. This is related to the northern location of Ganjingzi, and its reservoir and forest natural resources.

The results indicate that the most popular types of attractions in the research area are hydrographic landscapes, followed by biological tourism resources, and the least popular are geomorphologic landscapes and catering and shopping. Overall, natural resources-based attractions were rated 22 percentage points higher than humanistic attractions, accounting for $61 \%$ of the total. With the least popular scenic spots comprising food and shopping, Dalian 
has not formed a very regional commercial attraction. Therefore, we can draw a conclusion that the popularity of Dalian's attractions is further dependent on environmental development.

\subsubsection{Spatiotemporal distribution of the seasonality of scenic spots' heat}

The seasonality in this article is represented by the summer vacation and winter vacation periods, which are respectively from July to August and January to February. Fig. 3 highlights the seasonal spatial-temporal distribution of the scenic spots in the five districts. Many venues have been very popular in both summer and winter vacations, especially the representative scenic spots in Dalian: Xinghai Square, Tiger Beach Ocean Park, Shengya Ocean Park, Binhai Road, Lvshun Military Port, etc.; their popularity reaches its peak in the summer. However, after the middle of October, the lido and marine entertainment activities are closed seasonally, and outdoor performances at the Ocean Park and Forest Zoo are also closed due to rising sea-winds, as shown in Fig. 3(b). Although the historical heritage and cultural attractions in Lushunkou are open in winter, many people choose winter-appropriate recreational activities, such as skiing and hot springs.

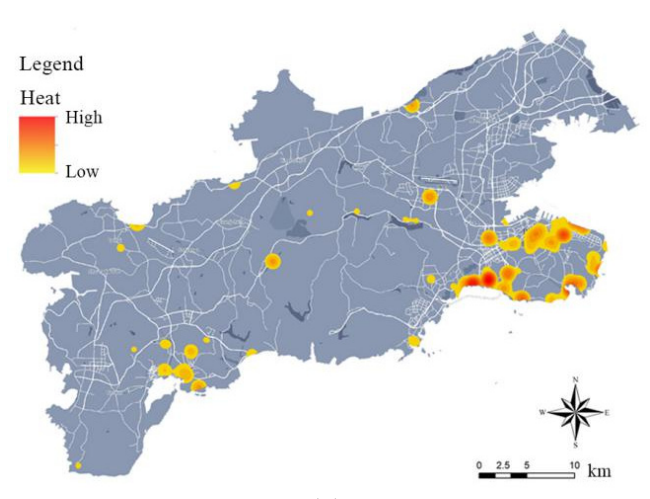

(a)

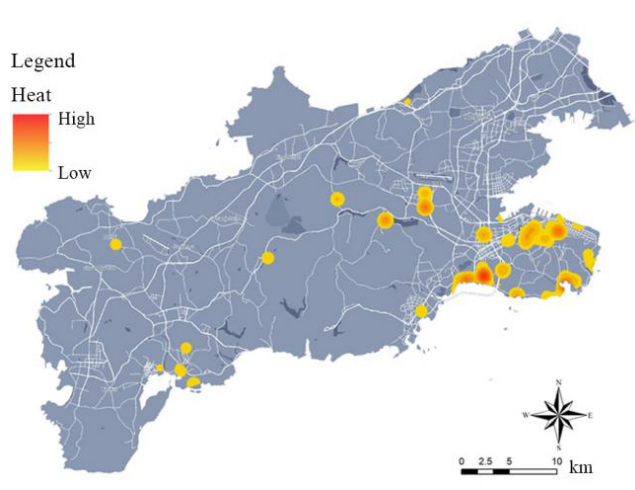

(b)

Figure 3: Heat of scenic spots in vacations. (a) Summer vacation; and (b) Winter vacation.

The representative tourist attractions in Dalian are mainly composed of seaside parks, city scenery, and cultural relics and historic sites in Lvshunkou, etc.; these essentially cater to the category of one-time tourists, and are very attractive to tourists from other places. The summer vacation is the peak season for attracting non-local visitors in Dalian, as the density of tourists changes due to climate. Above all, the change in the popularity of scenic spots between winter and summer is attributed to the characteristics of the type of tourism resources offered.

\subsubsection{Spatiotemporal distribution of scenic spots' heat in public holidays}

Since China began to implement the Public Holiday Strategy in 1999, the public holiday has evolved into a mini-break in the past ten years. These occasions include Golden Week, New Year's Day, and traditional Chinese festivals (e.g. Tomb-Sweeping Day, Dragon Boat Festival, Mid-Autumn Festival); the average length of each holiday is 4.2 days. The emergence of the national-scale concentrated holiday inevitably changed the previous forms of tourism. Most people choose short trips because the time is short, and there are more travel 
products for mini-breaks. With the continuous increase of private car ownership and the diversified development of scenic spots in Dalian, the weekend has become a concentrated period of hot spots.

The hottest and densest spots for mini-breaks and weekends are again the three eastern areas, especially the traditional attractions, and those in coastal areas (as shown in Fig. 4). However, places offering diversity and fashionable consumption are popular as well (e.g. Dalian Donggang Venice Water City, Momicafe Books Co, Fifteen Library Arts Centre). These hot spots, which have become emerging popular attractions in Dalian in recent years, are more inclined towards fashion culture and avant-garde arts, and have become Web celebrity places for young people. Fig. 4 illustrates the difference between the popularity of scenic spots in the mini-breaks and the weekend. The attractions in the three eastern districts were more popular on weekends; the scenic spots for mini-breaks are generally hotter and more widely distributed. In addition to the eastern region, Sanhuan Ranch, Tangli Lake Park, Yingge Stone Botanical Garden, Xishan Reservoir and other eco-tourism attractions have become popular destinations for citizens. Commercial attractions such as hot springs and other indoor entertainment facilities are very hot. It is difficult to distinguish the difference brought by seasonal changes in this section. However, the combination of the concentration and diversity of scenic spots with specific solar periods has formed a special tourism mode, due to the institutional nature of public holidays. For example, the cherry blossoms in Dragon King Pond almost coincide with Labour Day; the Yingge Stone Botanical Garden contains sub-attractions of different flowering periods; and in both the Dragon Boat Festival and the Mid-Autumn Festival, visitors can enjoy extraordinary scenery. Finally, the distribution of scenic spots heat in public holidays is more diversified during public holidays.

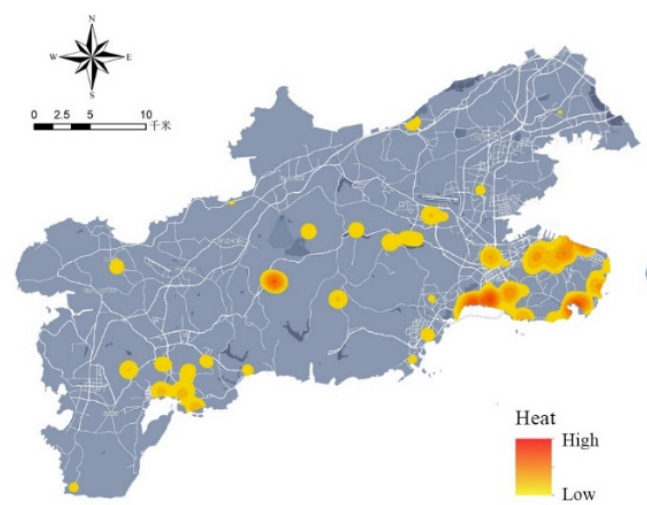

(a)

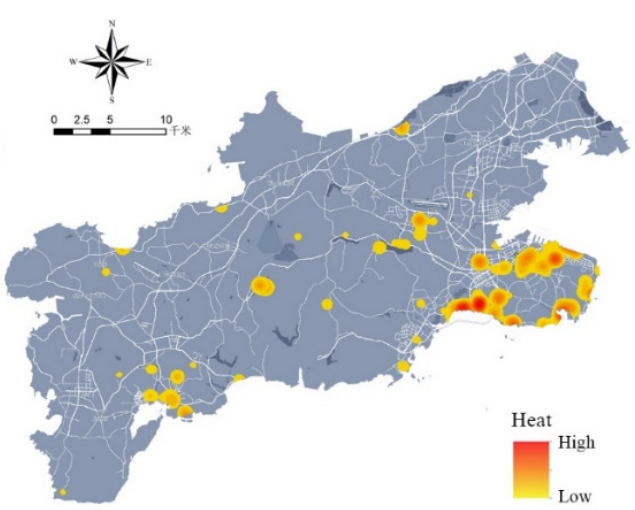

(b)

Figure 4: Heat of scenic spots in public holidays. (a) Mini-break; and (b) Weekend.

Eco-tourism resources are more attractive to residents than the typical scenic spots in Dalian, which reflects the originality of resource characteristics and the participation of tourists in tourism activities. This indicates that this type of tourism puts more emphasis on flexibility in time. Thus, the issue of the tourism market and the preservation and development of tourism resources should be given special consideration. 


\subsection{Street network configuration based on social issues}

In this section, we not only used spatial syntax, but also combined the regional population density and the spatial-temporal distribution of scenic spots on public holidays, in order to comprehensively analyse the current contradictions of the street network configuration.

Fig. 5 shows the regional population density; Shahekou has the highest, and the area with the lowest density is Lvshunkou. The distribution of heat and density of scenic spots are almost consistent with the distribution of population density. This illustrates that the development and diversification of scenic spots are related to population density and business development. Moreover, the heat distribution of scenic spots on the east and west sides is obviously unbalanced. This leads to the problem that inhabitants' journey to the tourist destinations becomes inconvenient, especially when large numbers of residents flow from the city centre in the east to popular attractions in the mid-west. Combined with Fig. 1 and Fig. 6, it can be seen that the traffic flow in the centre is very simplex under the influence of the terrain, so that the roads connecting the east and west scenic spots are particularly prone to congestion; especially the only route leading to the popular ecological scenic spots in Lvshunkou and Ganjingzi (Figs 5 and 6). Furthermore, many parents choose to take their children close to nature as a weekend activity; this has undoubtedly increased the space-time contradiction between local citizens' inflow and the natural resources scenic spots. As shown in Fig. 6, the peak of the global integration is concentrated in the east, spanning three regions from north to south. The population density and traffic accessibility do not coincide completely, and the popular scenic spots are excessively concentrated in the southeast. In contrast, the overall integration degree of the central and western regions is relatively low, and even the southern coast of Lvshunkou is close to the lowest value. The street network is mostly the proximal type, and the single spatial structure impairs accessibility, making it extremely inconvenient for tourists to flow in both north-south and east-west directions. The scenic spot of Lvshunkou is not ideal from the perspective of space composition, and the space attraction is weak. As a result, most inhabitants choose to travel nearby on the same day, which reduces the sense of tourism experience and aggravates the imbalance in the development of scenic spots.

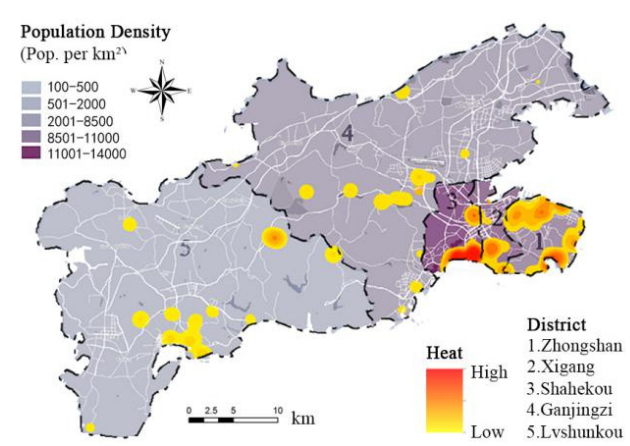

Figure 5: Population density and heat distribution of attractions in mini-holidays and weekends.

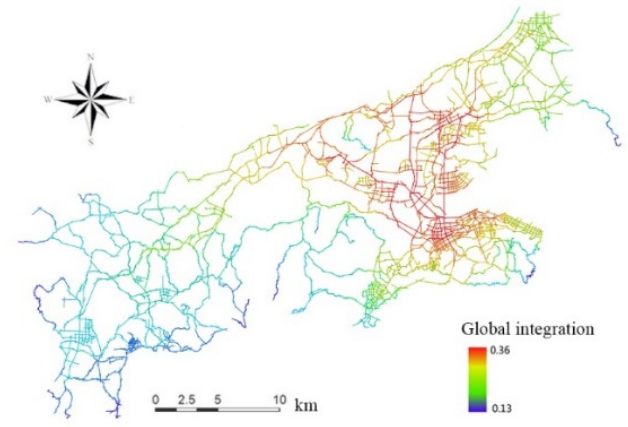

Figure 6: Analysis results for global integration in Dalian. 


\section{DISCUSSION AND CONCLUSIONS}

Summarizing the discussion in the above section, the results indicate that the attractiveness of the tourist attractions in Dalian is unbalanced, and the attraction for foreign tourists has gradually weakened. Meanwhile, the lack of sustainable planning for infrastructure construction has led to crowding of tourists and traffic congestion in the scenic area; moreover, the flow of inhabitants and tourists is contradictory. Based on the sustainable development framework, the specific optimization strategies are as follows.

\subsection{Economic aspects}

The development of Dalian's tourism industry should be consistent with the city's economic development goals. With the assistance of the brand effect of the hot spots, the marketing publicity should be strengthened to improve the overall attractiveness of the tourism system to foreign tourists. Different development modes should be adopted to avoid a shielding effect. Business circles should be more closely related to scenic spots. We should preserve and publicize the distinctive culture of Dalian from the perspective of business development, and attract young people by offering minority and avant-garde art tourism areas. In addition, the construction of cultural venues should be increased, to provide citizens with more cultural activities. The tourism industry should be combined with the culture and arts industry, to drive business development.

\subsection{Environmental aspects}

While satisfying the requirements of residents' trips in different periods, optimizing ecotourism is a necessary means of preserving the ecological environment. The tourist capacity of scenic spots should be effectively evaluated (e.g. ecological capacity, tourist psychological capacity, tourist social capacity and tourist economic capacity). When developing natural tourism resources, the ecological environment should be protected to avoid environmental degradation, and the tourism industry cannot continue to develop. Therefore, we should first formulate a plan to fully demonstrate, when developing ecotourism products, that tourists can do various activities within the prescribed route and scope. We should not focus only on economic benefits and ignore environmental benefits.

\subsection{Social aspects}

According to the unbalanced layout of scenic spots, it is necessary to build a convenient and unimpeded tourism transportation network; this should be constructed to strengthen the spatial relevance of various elements in the scenic spot system, and improve the scientific rationality of regional planning. Before the construction of a scenic area, it is required to scientifically predict the carrying capacity of tourists in that area, to infer the parameters related to traffic. Moreover, traffic jams can increase carbon emissions, which in turn have a negative impact on the environment. Therefore, it is necessary to scientifically, prospectively and reasonably plan scenic areas and roads, and improve the convenience of public transportation. Relevant departments should organically combine various lines of public transportation to serve popular attractions, to form a dual-track model for tourists and residents. For example, the initial establishment of the Xinghai Bay Bridge was intended to ease the traffic pressure on Zhongshan Road, but it has become a new landmark. It not only relieves traffic pressure, but also facilitates the coordinated development of scenic spots in the east and the west. 
Although we have discussed the issue of convenience travel during shorter holidays, due to the limitations of data collection, this article includes insufficient research on the contradiction between residential areas and scenic spots. However, the current research provides directions for research on the coordination between residents and non-local tourists, and lays the foundation for further construction that serves both the tourism industry and sustainable urban development. In conclusion, with the improvement of citizens' material and spiritual needs, the relationship between urbanization and tourism needs to be further balanced. It is necessary to pay attention to the common development of cities and tourism from the perspectives of environmental, economic and social development.

\section{REFERENCES}

[1] Shen, L.Y., Jorge Ochoa, J., Shah, M.N. \& Zhang, X., The application of urban sustainability indicators: A comparison between various practices. Habitat International, 35(1), pp. 17-29, 2011.

[2] Camagni, R., Sustainable urban development: Definition and reasons for a research programme. International Journal of Environment and Pollution, 10(1), pp. 6-26, 1998.

[3] Kaya, L.G., Application of collaborative approaches to the integrative environmental planning of Mediterranean coastal zone: Case of Turkey. Bartın Orman Fakültesi Dergisi, 12(18), pp. 21-32, 2010.

[4] Chen, L., Thapa, B., Kim, J. \& Yi, L., Landscape optimization in a highly urbanized tourism destination: An integrated approach in Nanjing, China. Sustainability (Switzerland), 9(12), 2017.

[5] Naramski, M. \& Herman, K., The development of mobile tourism in the upper silesian metropolitan area of Poland. Sustainability (Switzerland), 12(1), pp. 1-22, 2020.

[6] Chantre-Astaiza, A., Fuentes-Moraleda, L., Muñoz-Mazón, A. \& Ramirez-Gonzalez, G., Science mapping of tourist mobility 1980-2019. Technological advancements in the collection of the data for tourist traceability. Sustainability (Switzerland), 11(17), pp. 1-32, 2019.

[7] Yunxian, C., Renjie, L., Shuliang, Z. \& Fenghua, G., Measuring multi-spatiotemporal scale tourist destination popularity based on text granular computing. PLoS ONE, 15(4), 2020.

[8] Martí, P., García-Mayor, C. \& Serrano-Estrada, L., Taking the urban tourist activity pulse through digital footprints. Current Issues in Tourism, pp. 1-20, 2020.

[9] Zheng, Y.T., Zha, Z.J. \& Chua, T.S., Mining travel patterns from geotagged photos. ACM Transactions on Intelligent Systems and Technology, 3(3), 2012.

[10] van der Zee, E., Bertocchi, D. \& Vanneste, D., Distribution of tourists within urban heritage destinations: a hot spot/cold spot analysis of TripAdvisor data as support for destination management. Current Issues in Tourism, 23(2), pp. 175-196, 2020.

[11] Xiao, G., Juan, Z. \& Zhang, C., Travel mode detection based on GPS track data and Bayesian networks. Computers, Environment and Urban Systems, 54, pp. 14-22, 2015.

[12] Du, T., Vejre, H., Fertner, C. \& Xiang, P., Optimisation of ecological leisure industrial planning based on improved GIS-AHP: A case study in Shapingba District, Chongqing, China. Sustainability (Switzerland), 12(1), pp. 1-29, 2020.

[13] Li, Y., Abdel-Aty, M., Yuan, J., Cheng, Z. \& Lu, J., Analyzing traffic violation behavior at urban intersections: A spatio-temporal kernel density estimation approach using automated enforcement system data. Accident Analysis and Prevention, 141, 2020. 